\section{$\underset{\substack{\text { hommes } \\ \text { \& migrations }}}{ }$}

Hommes \& migrations

Revue française de référence sur les dynamiques

migratoires

$1314 \mid 2016$

Migrations chinoises et générations

\title{
Khaled Osman, La Colombe et le moineau
}

La Roque d'Anthéron, Vents d'ailleurs, 2016, 173 p., $20 €$

\section{Mustapha Harzoune}

\section{Q OpenEdition}

1 Journals

Édition électronique

URL : http://journals.openedition.org/hommesmigrations/3682

DOI : 10.4000/hommesmigrations.3682

ISSN : 2262-3353

\section{Éditeur}

Musée national de l'histoire de l'immigration

\section{Édition imprimée}

Date de publication : 1 avril 2016

Pagination : 178-179

ISBN : 978-2-919040-35-3

ISSN : $1142-852 X$

\section{Référence électronique}

Mustapha Harzoune, « Khaled Osman, La Colombe et le moineau », Hommes \& migrations [En ligne], 1314 | 2016, mis en ligne le 19 septembre 2016, consulté le 24 septembre 2020. URL : http:// journals.openedition.org/hommesmigrations/3682 ; DOI : https://doi.org/10.4000/ hommesmigrations.3682 


\section{LIVRES}

son vide assassin. Idem en Grèce - ì Aytilène ou Lesbos ou "la crise a tué un pays, pas les individus" ou ì Acqua formosa, ce village de calabre, "ressus eité" grâce ì l'aceueil de réfugiés. Des réfugiés, pas des clandestins, qui "ont le droit - le droit, pas la charité- de trou ver asile dans le pays d'Europe où its s'exilent." D'Athènes ì Ceuta, du Sinaï ì Ealais, Jean Paul Aari montre les consé quences de la (non) politique de l'Eu rope forteresse. II décrit les indignités des arrangements, avec l'ex ami Kha dafi par exemple. L'ttalie de Berlusconi et l'Europe s'accommodaient alors de l'horreur descamps de rétention libyens. Aujourd'hui, une autre traite négrière, à ta couleur de l'istam, se poursuit, sauva gement, en Libyeouen Égypte.

Ee livre, ì la fois reportage et scénari sation, raconte la vie de ceux dont on wache-les visages et les souffrances der rière des mots "exilés", "sans papiers" ou "inflltrés" (tsraël).Ces êtres de chairet de-sang se nomment Robiel, l'Érythréen -Cédric, le Eamerounais défenestré par ta police marocaine; Zachiel, l'imam afghan qui refuse de "pousser les gens ès'entretuer", Fassi,legamin de-Guinée qui rêve de foot; Rahwa, l'Érythréenne taissée pour morte dans une "mai son de torture" au Sinaï, Salomon, un des cing miraculés d'une embarcation de plastique qui a dérivé pendant 23 jours... Et "l'essentiel" n'est pas la guerre, les persécutions ou la faim: "On peut survive et même vivre dans la plupart des pays d'Afrique, du Aloyen Orient et d'Asie. (...) Hs saventcommentestleurvie et comment elle vase terminer. Tout est déjà tracé, sans espoir de changement. Pourchanger le destin, ne reste plusqu'à tout briser, partir, ouvrir le champ du possible mêmes'il peut mener ì l'enfer. Etils partent (..) poser une pierre dansle jardin d'un autre monde." M. H.

Samir, prof d'histoire de civilisation arabe à Paris, qui a coupé les ponts avec son passé, comprend qu'il s'agit d'Hicham, son frère jumeau, lequel lui demande de revenir avec Lamia, son ex petite amie. Commence alors l'enquête pour retrouver la jeune femme..

Samir est réveillé par un appel qui vient de la Place Tahrir au Caire. Un soignant lui apprend qu'on a trouvé son numéro de téléphone sur un homme qui vient d'être pris en charge. Avant de sombrer dans le coma, l'inconnu a pu articuler : "qu'il vienne mais avec elle".
Cette trame semble n'être qu'un subterfuge littéraire pour entretenir le lecteur de thèmes d'importance sur l'exil et sur la culture, la mémoire ou le devenir du monde "arabe". Il y a comme un écho aux Considérations sur le malheur arabe de Samir Kassir (Actes Sud, 2008). En érudit et éminent traducteur, l'auteur fait dialoguer les siècles et les textes, 
exhume de l'oubli la reine Zabba', cite et commente Darwich, Donkol ou Kanafani. Au centre, il y a comme un plaidoyer, le rappel d'une évidence : la civilisation arabo-musulmane renferme des "merveilles", linguistiques, littéraires, philosophiques et même, exégèse coranique oblige, religieuses, que seule l'ignorance a conduit à oublier. Les sujets et les références foisonnent. L'intelligence, sur le gril, s'agite à réfléchir à ce goût pour l'amertume et la tristesse de la poésie arabe, à la signification de "rahma", à l'échec des indépendances ou à questionner le mépris des élites, devenu "défiance" des intellectuels pour leur peuple...

L'autre et fragile procédé littéraire sur lequel reposent nombre de démonstrations est la brouille des deux frères. Elle tient à une dissertation, par leur père imposée, sur l'interprétation de l'expédition de Bonaparte. Samir qui avait lu les notes paternelles - ce qu'il traine comme une culpabilité - mit en avant l'intérêt culturel de l'entreprise quand Hicham dénonçait le mensonge consistant à "apporter la lumière à une population sous la menace des armes". Comme toute ressemblance avec l'actualité ne serait pas fortuite, Samir finira par reconnaître son erreur : "l'expédition avait trahi ses idéaux proclamés, ceux de la Révolution française...". Car, et c'est là l'autre objet de ce roman par trop didactique - au détriment des personnages et de l'intrigue -, il y a la francophilie d'une famille mise à rude épreuve. La chute est d'autant plus douloureuse, que l'on tombe de haut, la France incarnant les idéaux de liberté et de fraternité. Ainsi, Samir et Lamia seraient des étrangers transformés en "bêtes de foire". L'auteur rassemble pêle-mêle la déception de l'une, le "malaise" de l'autre et... les regards portés sur une femme voilée. Quoiqu'il en soit c'est d'intégrité dont il est question, une intégrité niée et fragmentée comme dans les peintures de Lamia. Mais la blessure de l'exil n'est pas sans ambiguïtés. La rupture de Samir avec l'Égypte est renvoyée à la "peur de mettre en danger le confort dont il jouissait en France, peur d'avoir à se battre pied à pied et non plus à distance". Et, pour "conserver la maîtrise de son destin", comme la reine Zabba', fautil rentrer en Égypte, se tenir au côté de ceux qui écrivent l'histoire, qui refusent d'être condamnés, comme dirait Driss Chraïbi, à "un autre avenir que notre passé " (Vu, lu, entendu, Denoël, 1998) ? M. H. 